the Service by taking a larger share of the N.H.S. cake.

I hear also that critics in the royal olleges have suggested that it is necessary for juniors to work every other night in order to get adequate training. I know that they cannot have done their homework; many juniors in recognized training posts have been working one night on in three for years; are they now to be on duty more often? The colleges have stated that the length of training may have to be increased; this will affect few juniors, most of whom stay much longer than the minimum time in the training grades because of poor career opportunities.

The D.H.S.S. had the opportunity to plead poverty at the time of the 1973 review, but this does not seem to have impressed Lord Halsbury when he made the award. Recently I have heard talk of the proposed changes not being implemented. The consequences of that do not bear consideration.-I am, etc.,

D. E. F. NEWTON

Wideopen,

Northumberland

\section{Jaundice after Halothane}

SIR,-We would like to support in general terms the various letters which urge caution in interpreting the findings reported by $\mathrm{Dr}$. W. H. W. Inman and Professor W. W. Mushin (5 January, p. 5) though appreciating that "reporting faults" were due to anaesthetists themselves. However, the unusual action of the Committee on Safety of Medicines in circulating anaesthetists drawing attention to the one published article could easily be taken as giving official backing to the views expressed by the authors. We note that the committee stated in their letter that "jaundice may ... follow the administration of halothane" and that "repeated exposure within a period of a few weeks may carry a greater risk" (our italics). Like many other groups of anaesthetists we know of cases of jaundice following anaesthesia and are aware of the possible effects of the agents.

It will be appreciated that halothane has been more extensively studied, both in the laboratory and in the clinical situation, than any other agent. Over the years it has proved to be one of the safest anaesthetic agents available. It is our opinion that halothane should still be used when it is considered the agent of choice in a particular instance and that, in these circumstances, even repeated administrations are fully justified in comparison with the disadvantages and risks of alternative techniques. In each individual case the overall benefit to patient and surgeon must be assessed in this light, and while appreciating the need for caution, in appropriate circumstanoes in our division we shall continue to use and teach the use of halothane when, in the opinion of the anaesthetist, it is the agent of choice.-We are, etc.,

JOHN DUNDEE Chairman,

R. C. GRAY R. C. GRAY
Vice-Chairman,

Royal Victoria Hospital, Anaesthetic Division

Reylfast
Assessing the Safety of Comatose and Postanaesthetic Patients

SIR,-Following head injury, poisoning, general anaesthesia, and other causes of coma patients are at risk from respiratory obstruction or depression. Assessment of the patient's safety is essential, and for this a test is required which will determine whether the patient is able to make the proper response to the likely hazards. Experience with patients recovering from anaesthesia has proved instructive and suggests that some methods in use are inappropriate and may be misleading.

Reoovery is usually well understood where anaesthesia has been maintained with an inhalation agent or by means of an intravenous agent such as a barbiturate. The patient progresses steadily from unconsciousness to a point where the anaesthetist or recovery nurse is satisfied that it is safe for the patient to return to the ward. Since the patient becomes progressively safer as time passes, the decision that recovery has occurred is not a critical one and current methods of assessment are satisfactory.

In contrast, the patient emerging from anaesthesia based on intravenous analgesics such as pethidine, together with a muscle relaxant, commonly responds and speaks in the operating theatre shortly after the conclusion of surgery. There is a natural tendency to regard such a patient as "recovered" and some of the commonly used methods of assessment may appear to confirm this. This is because they involve the application of a stimulus, either auditory or painful, which will in itself arouse the patient to a lighter, and hence safer, level of consciousness. However, the patient is in more danger when left undisturbed, so that any test relying on the application of a stimulus cannot be entirely valid.

The likely dangers are respiratory depression and respiratory obstruction, and the patient's ability to meet these hazards with the proper response may be assessed as follows. Instead of being stimulated the patient remains undisturbed for a few minutes and is allowed to breathe spontaneously with the head in a normal position. Gradually increasing downward pressure is then applied to the point of the chin. This will tend to flex the neck and depress the jaw towards the manubrium and is, in effect, the very reverse of the familiar manoeuvre employed to open the upper airway in an unconscious patient. Recovery is deemed to have taken place when the patient has reached that level of consciousness, and has the strength and co-ordination, necessary to overcome the airway obstruction produced by the depression of the jaw. Until the patient can respond in this way he should remain under close supervision.

We arrived at the technique independently after similar experiences. Four patients were encountered, each of whom awoke in the theatre after general anaesthesia for abdominal surgery. These patients had recovered sufficiently to obey commands and to speak spontaneously and oherently. However, all subsequently developed respiratory arrest, progressing in two cases to cardiac arrest. Fortunately, all were successfully resuscitated. In each case it had been assumed that the patient had "recovered" because of his ability to speak and obey instructions. Had these cases been assessed as described above the frightening and potentially fatal episodes could almost certainly have been avoided. Subsequent experience has supported this view; cases have occurred where, after apparent recovery, respiratory obstruction or depression has ensued in undisturbed patients or in those subjected to our chin-depression test.

Though the method was developed for use after anaesthesia and, in particular, after the intravenous - analgesic/muscle - relaxant technique, it has proved equally suitable for the assessment of any comatose patient. Moreover, it provides a most instructive demonstration of the particular risks to which such a patient is exposed. A nurse witnessing this test tends to appreciate the importance of her care of the patient much more than if she merely sees him respond to a stimulus.

Though similiar methods must be in use, we have not seen them reported, and discussion with colleagues indicates that their application is extremely limited. The technique described has proved useful to us and may be of interest to others.-We are, etc.,

A. W. GROGONO A. R. DEC. DEACOCK

Department of Anaesthesia,

Royal Free Hospital,

London N.W.3

\section{The Kidney in Infective Endocarditis}

SIR,-I was taught many years ago and still believe that the characteristic renal lesion in bacterial endocarditis is focal embolic nephritis. The largest fragments detached from cardiac vegetations cause gross infarction; the smallest obstruct vessels supplying or within individual glomeruli, which then eventually fibrose. The effect of this process can be seriously to impair renal function, without the co-existence of any other kind of nephritis. Hence my perplexity on reading the paper by Dr. J. M. Boulton-Jones and others (6 April, p. 11) entitled "Renal Lesions of Subacute Infective Endocarditis," in which lesions of this well-reoognized type are not mentioned and the word "embolism" is never used.

These authors' investigations and their arguments in favour of a different aetiology in their five cases are beyond my understanding, so that I am in no position to contest their conclusion. I am aware that a diffuse glomerulonephritis can also oocur in this disease, but is it not possible that some of the glomerular lesions described resulted from micro-embolization, and if so should not this possibility at least be admitted? In none of the case histories is there any reference to Osler's nodes, splinter haemorrhages, or the findings of retinosoopy. If any such lesions existed they must have had their counterpart in the kidney. Unless in the absence of all such signs, how can it be convincingly claimed that the renal lesions observed were of a wholly different nature?-I am, etc.,

Wokingham, Berks

LAWRENCE P. GARROD

\section{Pre-eclampsia and the Kidney}

SIR,-I have read with interest the evidence presented by Dr. O. M. Petrucoo and others (16 March, p. 473) suggesting that im- 
munological processes may play a part in the causation of pre-eclampsia, together with your leading article (p. 468). Curiously enough Dr. W. B. Patterson has just sent me his $b^{1}{ }^{1}$ in which he notes the association of fetal hyperplasia with late pregnancy toxaemia.

It has always amazed me that attempts are still being made in this cybernetic era to pinpoint one lesion as being the cause of, or always being associated with, preeclampsia and presumably eclampsia. Normal healthy life is entirely dependent on the adequate functioning of homoeostatic mechanisms and surely the wonder must be not that a relatively few pregnant women get pre-eclampsia, but rather that at least $85 \%$ of all pregnant women throughout the world can adjust to its demands. For consider that it is not yet understood why the mother does not immunologically reject the fetus, why her vascular system tolerates the vastly increased amounts of the steroid hormones, or how she overcomes the metabolic and mechanical stresses and strains of pregnancy. It is only necessary to recall the effects of hormonal stimulation on the spiral arteries during the menstrual cycle to recognize the potential vulnerability of small vessels to excessive hormonal influence.

The symptoms associated with "glomerular endotheliosis" are clinically indistineuishable from those associated with chronic glomerular nephritis. So much for precise lesions. Further, the multigravida rarely manifests hypertension, proteinuria, and oedema unless she had these symptoms during her first pregnancy and yet electron microscopical studies of material removed at renal biopsy from 152 multiparae revealed no detectable lesion or renal abnormality in half the cases. ${ }^{2}$ It would appear to follow that "glomerular endotheliosis" is a lesion which is most commonly seen in first pregnancies, clears up completely, and is unlikely to recur in subsequent pregnancies, even though the signs and symptoms of pre-eclampsia may. Then too, in another leading article in the same issue (p. 470), you draw attention to the fact that it is not at present known "whether autorepulation oocurs in essential hypertension and, if it does, whether it can acoount for the variations in plasma volume."

The main object of pregnancy is to produce a viable, healthy child, and if its nutrition is at stake, whether because of defective kidneys or an inadequate placenta, feed-back mechanisms are called into play and, in extreme cases, provoke undesired svmptoms and lesions. MoCartney² does not think that glomerular endotheliosis causes pre-eclampsia and neither do Dixon and Robertson $^{3}{ }^{4}$ or Brosens, ${ }^{5}$ regard atherosis of the spiral arteries in the placental bed as a causal factor. Incidentally, Robertson observed during the course of conversation that the closest approximation he had seen to the vascular changes he had observed in the blood vessels of the placenta bed were those occurring in syphilitic lesions. It is consequently reasonable to observe that the lesions resulting from a break-down of a homoeostatic mechanism, whether in the liver, kidney, placenta, or brain, may not always be identical and conversely that apparently identical lesions may be produced in different ways.

Eclampsia is a syndrome and consequently no single cause for it will ever be dis- covered. The fact remains that whereas there were over 400 maternal deaths from pregnancy toxaemia in England and Wales during 1934, there were probably not more than four last year. This fall is due to improved antenatal care (including antenatal nutrition and rest) and the induction of labour at about 38 weeks in patients with hypertension. This was made possible only by the discovery of the antibiotics and by the introduction of the oxytocin drip. It is suggested that most of the women who get pre-eclampsia in this country had defects, whether detectable or occult, before pregnancy started. The ultimate control of both the hormonal and autonomic systems is vested in the hypothalamus and this control is largely determined in extent by the integrity of the vascular system, the endocrine organs, and the kidneys.-I am, etc.,

\section{Eastbourne}

G. W. THEobald

1 Patterson, W. B., Fetal Adrenal Hyperplasia; Its Relationship to Late Toxemia. Published by the Re!ationship

2 MoCartney, C. P., Gynaecologia, 1969, 167, 214. Dixon, H.' G., and Robertson, W. B., Fournal of Obstetrics and Gynaecology of the British Empire, $1958,65,803$

4 Dixon, H. G., and Robertson, W. B., Pathologia Dixon, Microbiologia, 1961, 24, 622.
et Gynaecology of the British Commonwealth, 1964, 71, 222 .

SIR,-Further to the interesting article by Dr. O. M. Petruoco and others (16 March, p. 473), I would like to report the results of some immunofluorescent antibody studies performed using placentae, maternal sera, and human kidney. The latter was removed from a 32-year-old parous patient shortly after a fatal accident. Histological assessment revealed the renal tissues to be entirely free of any disease, and as far as could be determined the patient had had no previous history of renal disease. Maternal serum was collected from pregnant patients at delivery who had had a normal antenatal period and from patients with preeclampsia. A section of the placenta was also obtained after each delivery. Sections $4 \mu \mathrm{m}$ thick of placenta and renal tissue were used for immunofluorescent antibody study, fluorescein-labelled anti-IgG, -IgA, and -IgM being used for staining purposes. Specificity of the antisera was checked by "blocking" studies.

Immunofluorescent staining without prior incubation with the respective maternal serum revealed no evidence of antibody deposition. But if incubation with maternal serum was performed before the immunofluorescent staining some interesting results were obtained. In the normal patients no specific fluorescence was seen either in the placenta or renal tissues. But in parients with pre-eclampsia marked IgM deposition was noted in the fetal capillaries in the placenta and in the proximal renal tubular epithelium, but none in Bowman's capsule. Moreover, if serum from a normal patien was incubated with a placenta from a toxaemic patient no specific fluorescence was seen. If serum from a toxaemic patient was incubated with tîsues from a normal patien the areas of IgM deposition were demonstrated as described earlier. IgG deposition was found in similar areas but was very much less dense.
These findings would support Curzen's ${ }^{1}$ concept of the presence of trophoblastspecific antibodies in patients with preeclampsia which cross-reacted with kidney tissues. From the above evidence they appear to localize in the proximal renal tubule. This part of the tubule is responsible for $\mathrm{Na}+, \mathrm{Cl}-, \mathrm{HCO}_{3}-$, and $\mathrm{H}_{2} \mathrm{O}$ homoeostasis, and if damaged could disturb the balanoe, possibly leading to some of the changes noted clinically in pre-eclampsia. How this "autoimmune" process is triggered is difficult to determine. Presumably there is a breakdown in the "barrier" protecting the fetus from rejection by the mother.

These findings, in conjunction with those reported by Dr. Petrucco and his colleagues namely, the constant finding of IgM, IgG, and complement deposition together in the glomerulus and its vascular supply-would lend support to the hypothesis that an immunological process plays a part in preeclampsia.-I am, etc.,

T. E. COCHRAN

Gynaecological Department,

City General Hospital,

1 Curzen, P., Fournal of Obstetrics and Gynaecology of the British Commonwealth, 1968, 75, 1128.

\section{Vaccination of Smallpox Contacts}

SIR, - We cannot allow to pass unchallenged the views on the control of smallpox expressed by Dr. D. J. Bauer (23 March, p. 576). All concerned with the management of smallpox know that vaccination during incubation cannot be relied on to protect, but it does not follow that it is without value. There is good evidence that sucoessful primary vaccination in the incubation period will modify the severity of the attack and reduce mortality by half. ${ }^{1}$ The effect on the incidence of smallpox is less crtain, but Dr. Bauer quotes a $56 \%$ attack rate in unvaccinated primary contacts who remained unvaccinated as against a $19 \%$ rate in previously unvaccinated contacts who received vaccination after diagnosis of the index cases. Active immunization by vaccination has been the first-line defence against smallpox for many years and has not only proved successful in eradicating the disease from endemic areas but has also succeeded in containing infection when reintroduced. This is a matter of such importance that it is essential to consider the evidence with the utmost care before adopting passive immunization and chemoprophvlaxis as the optimum protection for contacts.

The much-quoted trial by Kempe et al. in 1956 on the prophvlactic value of hyperimmune gammaglobulin had serious defects, but a subsequent trial in $1961^{3}$ produced more convincing evidence of the value of this procedure in conjunction with vaccination. The position with the thiosemicarbazone group of drugs is more uncertain. The trial of methisazone by Bauer et al., 4 which suggested a fourfold reduction in incidence, is open to criticism. The treatment and control groups were not allocated at random, those refusing treatment being assigned to the control group, and supervision of contracts was inadequate. A further trial of this compound in variola minor by do Valle et al..$^{5}$ supported Dr. 